\title{
Measuring and controlling the chaotic motion of profits
}

\author{
Cristina Januário ${ }^{1}$, Clara Grácio ${ }^{2}$, Diana A. Mendes ${ }^{3}$ and Jorge Duarte ${ }^{4}$ \\ ${ }^{1}$ ISEL-Instituto Superior de Engenharia de Lisboa, \\ Department of Chemistry, Mathematics Unit, \\ Rua Conselheiro Emídio Navarro, \\ 1949-014 Lisboa, Portugal \\ E-mail: cjanuario@deq.isel.ipl.pt \\ ${ }^{2}$ Department of Mathematics, Universidade de Évora, \\ Rua Romão Ramalho, 59, 7000-585 Évora, Portugal \\ E-mail: mgracio@uevora.pt \\ ${ }^{3}$ Department of Quantitative Methods, \\ Instituto Superior de Ciências do Trabalho e da Empresa, \\ Avenida das Forças Armadas, 1649-026 Lisboa, Portugal \\ E-mail: diana.mendes@iscte.pt \\ ${ }^{4}$ ISEL-Instituto Superior de Engenharia de Lisboa, \\ Department of Chemistry, Mathematics Unit, \\ Rua Conselheiro Emídio Navarro, \\ 1949-014 Lisboa, Portugal \\ and CIMA-UE, Universidade de Évora \\ E-mail: jduarte@deq.isel.ipl.pt
}

(Dated: February 9, 2009)

\begin{abstract}
The study of economic systems has generated deep interest in exploring the complexity of chaotic motions in economy. Due to important developments in nonlinear dynamics, the last two decades have witnessed strong revival of interest in nonlinear endogenous business chaotic models. The inability to predict the behavior of dynamical systems in presence of chaos suggests the application of chaos control methods, when we are more interested in obtaining regular behavior. In the present article we study a specific economic model from the literature. More precisely, a system of three ordinary differential equations gathering the variables of profits, reinvestments and financial flow of borrowings in the structure of a firm. Firstly, using results of symbolic dynamics, we characterize the topological entropy and the parameter space ordering of kneading sequences, associated with one-dimensional maps that reproduce significant aspects of the model dynamics. The analysis of the variation of this numerical invariant, in some realistic system parameter region, allows us to quantify and to distinguish different chaotic regimes. Finally, we show that complicated behavior arising from the chaotic firm model can be controlled without changing its original properties and the dynamics can be turned into the desired attracting time periodic motion (a stable steady state or into a regular cycle). The orbit sabillization is illustrated by the application of a feedback control technique initially developed by Romeiras et al. [1]. This work provides another illustration of how our understanding of economic models can be enhanced by the theoretical and numerical investigation of nonlinear dynamical systems modelled by ordinary differential equations.
\end{abstract}

PACS numbers: 89.65.Gh, 89.70.Cf, 89.75.Fb, 05.45.Ac

\section{INTRODUCTION}

One of the main subjects in economic dynamics is concerned with the explanation of the nature of fluctuations in economy. Most economic variables, such as gross domestic product, interest rates, production, stock prices as well as profits, investments and financial flow of borrowings, exhibit fluctuations over time: for instance these vary from fairly regular business cycles to very irregular fluctuations.

Inspired from chaos theory, economists (see [2] and [3]) started looking for nonlinear models generating erratic time series similar to the patterns observed in real business cycles. This search led to new nonlinear systems within the paradigm of optimizing behavior and perfectly competitive markets, generating chaotic business fluctuations.
A work on nonlinear dynamics and chaos may prompt us to ask a preliminary question: why should economists be mindful of chaos? A compelling and plausible reason to apply the chaos theory is due to the fact that chaos is no way a rare or pathological occurrence and the obtained results may be both mathematically sound and economically relevant.

The existence of deterministic dynamical systems with chaotic or 'strange' behavior has been known for over a century. The first discoverer of chaos was Henri Poincaré, in 1890, while studying the three-body problem. He found that simple, nonlinear, low-dimensional dynamical systems could exhibit very complicated behavior Despite initial insights in the first half of the $20^{t h}$ century, the theory of chaos became formalized 
only after mid-century. In fact, the idea of chaos remained dormant for several years, in spite of some fundamental contributions to the field such as those made by Birkhoff, Cartwright and Littlewood. The main catalyst for the development of chaos theory was made possible by the availability of electronic computers. A primary example of the use of electronic computers was the work of Lorenz, in the early sixties, whose interest in chaos came about accidentally through his studies in the context of a numerical investigation of a model of atmospheric turbulence. The electronic computation allowed the 'step-by-step' numerical integration of the differential equations involved in the model. Some of the important contributors to this new research field were Smale, Ruelle and Takens who provided significant theoretical results on chaotic dynamics and 'strange' attractors. Other relevant studies were produced both in the ergodic and in the geometric theory of chaos by, among others, Kolmogorov, Arnold, Anasov, Silnikov and Sinai. Currently, chaos theory continues to be a very active research area involving many different disciplines. A general view about the chaos theory history is given in the fascinating book [4] by James Gleik. The reader can also find in [5] fundamental mathematical concepts and results in the nonlinear science or theory of chaos.

The application of chaos theory to the analysis of a web of complex interactions among economic structures is particularly relevant and has generated extensive research programs. With the purpose of understanding this complexity, it is important to carefully study basic financial structures. In this context, the detailed examination of the chaotic behavior of dynamical variables involved in the financial structure of a firm, is accepted as critical to the study of important economic systems.

In order to isolate essential aspects of a firm dynamics, S. Bouali proposed in [6] a system of ordinary differential equations, extremely rich in complex dynamics, that contains just three dynamical variables: profits, reinvestments and financial flow of borrowings. With a nonlinear relation of reinvestments, the numerical simulations carried out in [6] suggest that the debt (i.e., the financial flow of borrowings) injects perturbation into the profit motion and seems to act as a chaos generating mechanism.

In recent years, there has been a considerable research effort into the analysis of chaotic systems. For instance, control, targeting, synchronization and forecasting of chaotic motion have proved well established results in the fields of applied mathematics, economy, physics and engineering. In particular, since the publication of the seminal paper of Ott, Grebogi and Yorke in $1990([7])$, there has been a great deal of progress in the development of techniques for the control of chaotic phenomena, with applications, for example, to economy, biochemistry, cardiology, communications, physics laboratories and turbulence. The central question addressed in the theory of chaos control is: given a chaotic system, how can we obtain improved performance and achieve a desired attracting time-periodic motion by making small controlling temporal perturbations in an accessible system parameter? ([1]).

In the context of economy, practical methods of this new and exciting field can be applied to show that the presence of chaotic motion in economic process does not necessarily need to be interpreted as a curse for economic theory and economic policy ([8]). Particularly, in order to control economic chaotic motion, we do not need to change the fundamental characteristics of the system, we just have to impose upon the dynamics some small perturbations. The application of small external perturbation to the model leaves the main features unchanged and is able to eliminate large business cycles.

The aim of the present article is to provide a contribution for the detailed analysis of the chaotic behavior of the Bouali model through the introduction, and comprehensive study of one-dimensional maps associated to the system, in terms of symbolic dynamics theory. In fact, we can gain some fundamental qualitative insights about the principles and mechanisms underlying chaotic behavior by studying low-dimensional maps, that incorporate representative dynamical properties of the system's attractor.

A quantifier for the complex orbit structure - an attribute used to define chaos - is the topological entropy. This attribute of chaos can be efficiently used to perform chaos control strategies. We also exhibit an application of the pole placement control technique, initially proposed by Romeiras et al. ([1]) as an extension of the OGY method (due to Ott, Grebogi and Yorke) carried out in [7]. In their work, these authors emphasize the fact that a chaotic attractor typically has embedded densely within it an infinite number of unstable periodic orbits. With small controlling perturbations to the system, the aim of this process is not to create new orbits with very different properties from the already existing ones, but to exploit the existing unstable periodic orbits in the absence of control. The control method will be applied considering the discrete time system obtained from the induced dynamics on a Poincaré section in the neighborhood of the desired periodic orbit. This technique involves the construction of a stabilizing linear map, obtained from a least squares fitting, using the Poincaré section points.

\section{DESCRIPTION OF THE MODEL}

The Bouali firm model is written in first-order autonomous differential equations and explores the dynamics of three endogenous variables: profits $(P)$, reinvestments $(R)$ and financial flow of borrowings $(F)$. In the next lines, we provide some details, established in [6], of each differential equation. 
(a) Profits equation

The reinvestments $R$ and the flow of borrowings $F$ are at the origin of profit creation. Therefore,

$$
\frac{d P}{d t}=\frac{1}{v}(R+F),
$$

where the coefficient $1 / v$ represents the rate of profits.

\section{(b) Reinvestments equation}

The reinvestments are made up of a fraction of profits according to the proportion $m$ and of the capitalization of reinvestments which are reassessed yearly at rate $n$,

$$
\frac{d R}{d t}=m P+n\left(1-P^{2}\right) R
$$

In this relation, when the profit reaches the value 1 , then the nonlinear part $\left(1-P^{2}\right)$ becomes null and the yearly reinvestments shall remain at a constant trend $m$. The remainder of the profits as well as the capital added value are distributed as dividends. Indeed, so long as the profit doesn't reach the unity threshold, the profits will be reinjected into the financing circuit of the firm. On the other hand, beyond the profit threshold $(P>1)$, the great movement of dividend distribution reduces the capitalization.

\section{(c) Flow of borrowings equation}

The firm can choose an increase of its capital by borrowing according to the debt rate $s$ proportional to selffinancing and by deducting a fraction of profits according the interest rate $r$, that is

$$
\frac{d F}{d t}=-r P+s R
$$

Gathering the previous information, we obtain the system

$$
\begin{aligned}
& \frac{d P}{d t}=\frac{1}{v}(R+F) \\
& \frac{d R}{d t}=m P+n\left(1-P^{2}\right) R \\
& \frac{d F}{d t}=-r P+s R
\end{aligned}
$$

with $P, R, F$ the dynamical variables in study and $v, m$, $n, r, s$ the parameters $(v \geq 1, m>0, n>0,0.05 \leq r \leq$ 0.1 and $0.2 \leq s \leq 0.4)$.

It is important to emphasize that, in a real economic context, the heuristic Bouali system can be used to analyze the asymptotic behavior of the financial dynamics of a firm, where the units of time are chosen so that time could be anything from hours to days. As we will see below, the interpretation given to the model dynamics is in harmony with the established timescales.

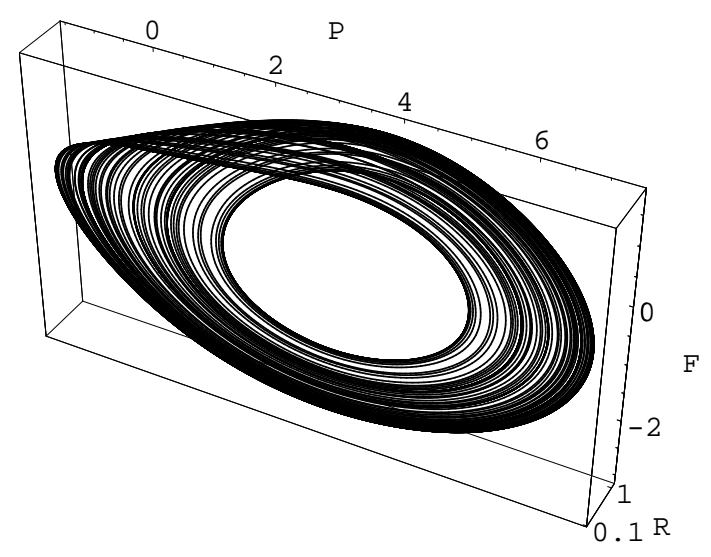

FIG. 1: Solution visualized as a trajectory in the threedimensional space for $r=0.068$ and $s=0.366$.

\section{UNIMODAL MAPS. SYMBOLIC DYNAMICS, TOPOLOGICAL ENTROPY AND CHAOS}

As we mentioned before, this article aims to introduce and carefully study a family of one-dimensional maps which allows us to characterize, in terms of symbolic dynamics theory, representative chaotic features of the Bouali firm model. For numerical investigation we will use throughout $v=4.0, m=0.04, n=0.02$ and consider $r$ and $s$ as control parameters. As we saw earlier, the parameter $r$ is an interest rate and the parameter $s$ is a debt rate $(0.05 \leq r \leq 0.1$ and $0.2 \leq s \leq 0.4)$.

Using numerical integration of the system (1), we can gain some insights about the geometry of the trajectories in the long run. After an initial transient, a structure emerges when the solution $(P(t), R(t), F(t))$ is visualized as a trajectory in three-dimensional space (Figure 1). Some projections of the three-dimensional trajectory onto a two-dimensional plane are exhibited in Figure 2 and in Figure 3

A comprehensive study of large-dimensional systems usually involves the evaluation of the possibility to perform their one-dimensional discrete-time representation.

With the purpose of understanding the main features of our three-dimensional flow, it is possible to construct one-dimensional maps recording the successive relative (local) maxima of the numerical solution $P(t)$, which represents the profits (see Figure 4).

These iterated maps consists of pairs $\left(P_{n}, P_{n+1}\right)$, where $P_{n}$ denotes the $n^{t h}$ local maximum. As shown in Figure 5 , the data from the chaotic time series appear to fall on a logistic curve. Indeed, treating the graph as a function $P_{n+1}=f\left(P_{n}\right)$ allow us to reveal particularly interesting features about the dynamics on the attractor. It is important to notice that, clearly, there are no a priori reasons for there to be any definite unidimensional relation between such consecutive values. The one-dimensional behavior is one of the noteworthy and eye-catching fea- 


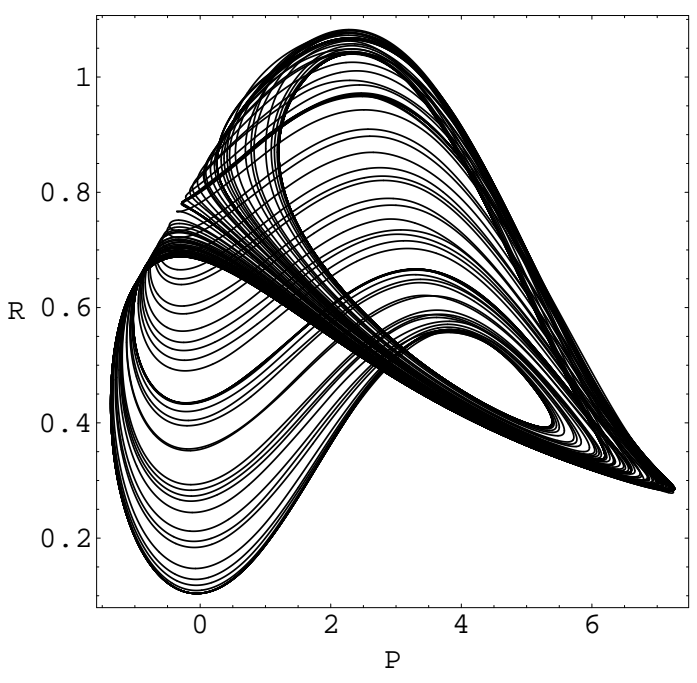

FIG. 2: Projection of the three-dimensional trajectory onto the $P R$-plane for $r=0.068$ and $s=0.366$.

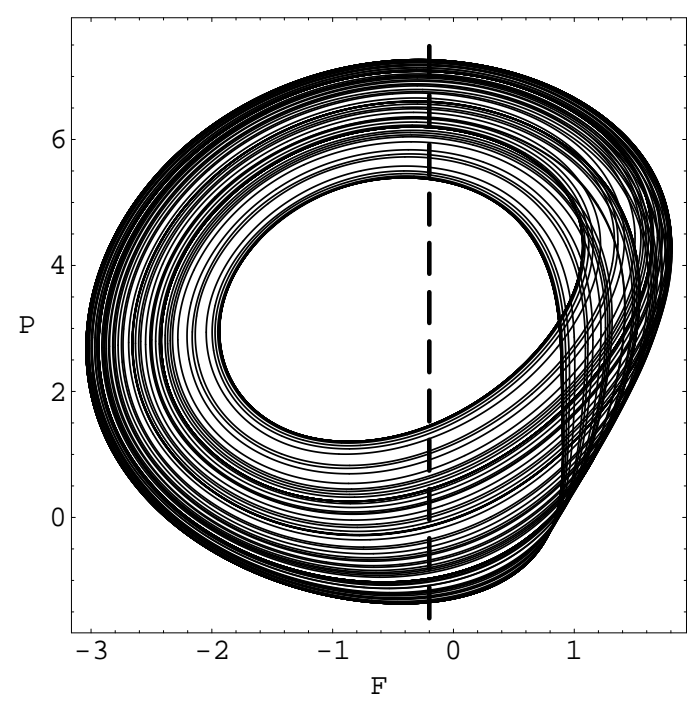

FIG. 3: Projection of the three-dimensional trajectory onto the $F P$-plane for $r=0.068$ and $s=0.366$. The meaning of the dashed line is assigned below.

tures of the dynamics.

The obtained iterated maps dynamically behave like a unimodal map, that is, continuous map on the interval with two monotonic subintervals and one turning point. In order to see the long time behavior for different values of the control parameters $s$ and $r$ at once, we plot typical bifurcation diagrams (see Figure 6 and Figure 7). An increase of the parameter values induces period-doubling cascade in the dynamics of profits. We can observe that for $s$ increasing the profits are increasing too (Figure 6) and when the interest rate $r$ is growing the profits are decreasing (Figure 7). Insofar, these numerical simulations strongly suggest that the solutions of the model may indeed be chaotic for a large region of the parameter space.

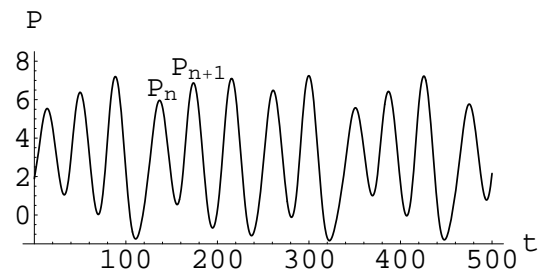

FIG. 4: Time sequence of profits for $r=0.068$ and $s=0.366$.

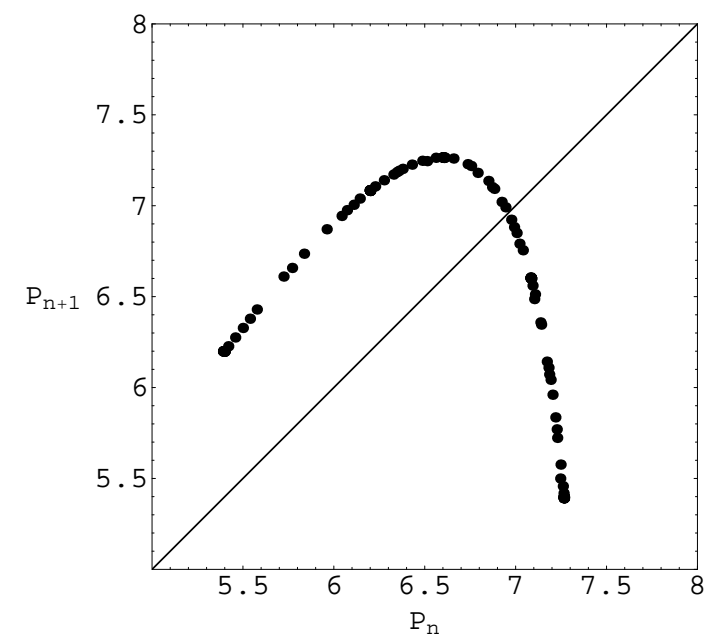

FIG. 5: The iterated map constructed from the successive local maxima of profit values $(r=0.068$ and $s=0.366)$.

At this point, we are in position to devote our attention to the study of the topological entropy of the logistic-like maps using results of symbolic dynamics theory.

The techniques and ideas of symbolic dynamics have found significant applications. One simplification in the study of dynamical systems is to discretize time, so that the state of the system is observed only at discrete ticks of a clock, like a motion picture. This leads to the study of the iterates of a single transformation. The theory of symbolic dynamics arose as an attempt to study systems

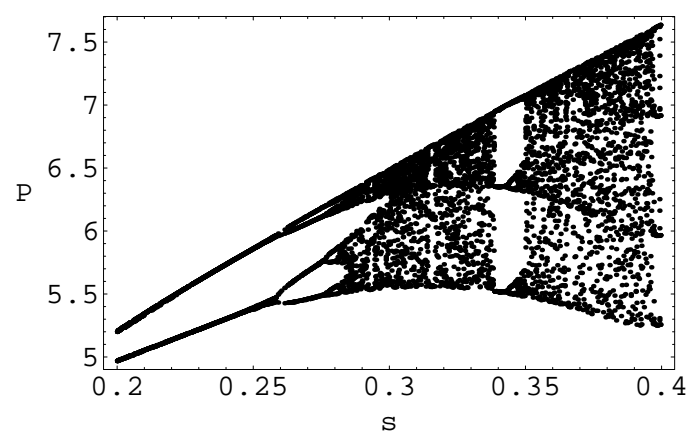

FIG. 6: Bifurcation diagram for $P$ as a function of $s$, with $r=0.068$ and $s \in[0.2,0.4]$. 


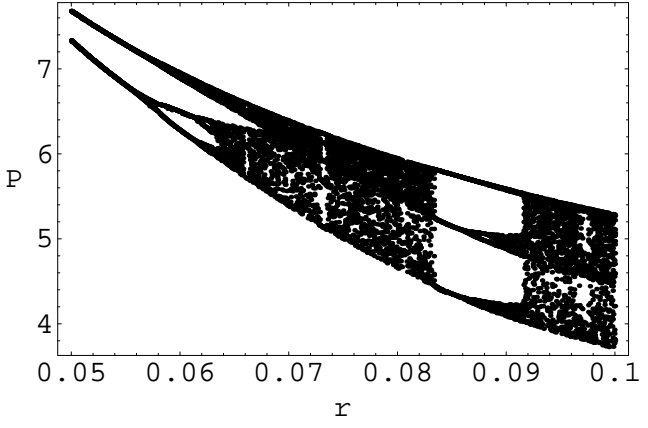

FIG. 7: Bifurcation diagram for $P$ as a function of $r$, with $s=0.3$ and $r \in[0.05,0.1]$.

by means of discretizing space as well as time. The idea is to divide up the set of possible states into a number of pieces, and keep track of which piece the state of the system lies in at every tick of the clock. Each piece is associated with a symbol, and in this way the evolution of the system is described by a sequence of symbols. This leads to a symbolic dynamical system that helps us to understand the dynamical behavior of the original system. The paper of Milnor and Thurston [9] sets up an effective method for describing the qualitative behavior of the successive iterates of a piecewise monotonic mapping.

In what follows, we apply techniques of symbolic dynamics, in particular some results concerning to Markov partitions associated with unimodal maps. For more details see [9], [10] and [11].

A unimodal map $f$ on the interval $I=[a, b]$ is a 2piecewise monotone map with one critical point $c$. Thus $I$ is subdivided into the following sets:

$$
I_{L}=\left[a, c\left[, \quad I_{C}=\{c\}, \quad I_{R}=\right] c, b\right],
$$

in such way that the restriction of $f$ to interval $I_{L}$ is strictly increasing and the restriction of $f$ to interval $I_{R}$ is decreasing (see Figure 5). Each such maximal intervals on which the function $f$ is monotone is called a lap of $f$, and the number $\ell=\ell(f)$ of distinct laps is called the lap number of $f$. Starting with the critical point of $f, c$ (relative extremum), we obtain the orbit

$$
O(c)=\left\{x_{i}: x_{i}=f^{i}(c), i \in \mathbb{N}\right\} .
$$

With the purpose of studying the topological properties, we associate to the orbit $O(c)$ a sequence of symbols, itinerary $(i(x))_{j}=S=S_{1} S_{2} \ldots S_{j} \ldots$, where $S_{j} \in \mathcal{A}=$ $\{L, C, R\}$ and

$$
\begin{array}{lll}
S_{j}=L & \text { if } & f^{j}(x)<c \\
S_{j}=C & \text { if } & f^{j}(x)=c \\
S_{j}=R & \text { if } & f^{j}(x)>c .
\end{array}
$$

The turning point $c$ plays an important role. Since the dynamics of the interval is characterized by the symbolic sequence associated to the critical point orbit. When
$O(c)$ is a $k$-periodic orbit, we obtain a sequence of symbols that can be characterized by a block of length $k$, the kneading sequence $S^{(k)}=S_{1} S_{2} \ldots S_{k-1} C$.

We introduce, in the set of symbols, an order relation $L<C<R$. The order of the symbols is extended to the symbolic sequences. Thus, for two of such sequences $P$ and $Q$ in $\mathcal{A}^{\mathbb{N}}$, let $i$ be such that $P_{i} \neq Q_{i}$ and $P_{j}=Q_{j}$ for $j<i$. Considering the $R$-parity of a sequence, that is the odd or even number of occurrence of a symbol $R$ in the sequence, than if the $R$-parity of the block $P_{1} \ldots P_{i-1}=$ $Q_{1} \ldots Q_{i-1}$ is even we say that $P<Q$ if $P_{i}<Q_{i}$. And if the $R$-parity of the same block is odd, we say that $P<Q$ if $P_{i}>Q_{i}$. If no such index $i$ exists, then $P=Q$.

The ordered sequence of elements $x_{i}$ of $O(c)$ determines a partition $\mathcal{P}^{(k-1)}$ of the interval $I=$ $\left[f^{2}(c), f(c)\right]=\left[x_{2}, x_{1}\right]$ into a finite number of subintervals labeled by $I_{1}, I_{2}, \ldots, I_{k-1}$. To this partition we associate a $(k-1) \times(k-1)$ transition matrix $M=\left[a_{i j}\right]$ with

$$
a_{i j}=\left\{\begin{array}{lll}
1 & \text { if } I_{j} \subset f\left(I_{i}\right) \\
0 & \text { if } I_{j} \varsubsetneqq f\left(I_{i}\right)
\end{array} .\right.
$$

Now we consider the topological entropy. As we pointed out before, this important numerical invariant is related to the orbit growth and allows us to quantify the complexity of the dynamics. It represents the exponential growth rate for the number of orbit segments distinguishable with arbitrarily fine but finite precision. In a sense, the topological entropy describes in a suggestive way the total exponential complexity of the orbit structure with a single number.

A definition of chaos in the context of onedimensional dynamical systems states that a dynamical system is called chaotic if its topological entropy is positive. Thus, the topological entropy can be computed to express whether a map has chaotic behavior, as we can see in [12] and [13]. In these references, Glasner and Weiss, in a discussion of Devaney's definition (of chaos), proposed positive entropy as a strong property for the characterization of complex dynamical systems, more precisely, as the essential criterium of chaos. Important results were constructed using this property (please see [14] and [15]).

The topological entropy of a unimodal interval map $f$, denoted by $h_{t o p}(f)$, is given by

$$
h_{\text {top }}(f)=\log \lambda_{\max }(M(f))=\log s(f),
$$

where $\lambda_{\max }(M(f))$ is the spectral radius of the transition matrix $M(f)$ and $s(f)$ is the growth rate,

$$
s(f)=\lim _{k \rightarrow \infty} \sqrt[k]{\ell\left(f^{k}\right)}
$$

of the lap number of $f^{k}\left(k^{t h}\right.$-iterate of $\left.f\right)$ (see [9], [16] and $[17])$. In summary, for each value of the parameter, the computation begins with the symbolic codification of the critical point orbits which determines a Markov 
partition of the interval. Then we compute the transition matrix associated with the Markov partition. Finally, the topological entropy is given by the logarithm of the highest eigenvalue of this transition matrix.

In order to illustrate the outlined formalism about the computation of the topological entropy, we discuss the following example.

Example 1 Let us consider the map of Figure 5. The orbit of the turning point defines the period-5 kneading sequence $(R L L R C)^{\infty}$. Putting the orbital points in order we obtain

$$
x_{2}<x_{3}<x_{0}<x_{4}<x_{1} .
$$

The corresponding transition matrix is

$$
M(f)=\left[\begin{array}{llll}
0 & 1 & 1 & 0 \\
0 & 0 & 0 & 1 \\
0 & 0 & 1 & 1 \\
1 & 1 & 0 & 0
\end{array}\right]
$$

which has the characteristic polynomial

$$
p(\lambda)=\operatorname{det}(M(f)-\lambda I)=1-\lambda-\lambda^{2}-\lambda^{3}+\lambda^{4} .
$$

The growth number $s(f)$ (the spectral radius of matrix $M(f))$ is $1.72208 \ldots$. Therefore, the value of the topological entropy can be given by

$$
h_{\text {top }}(f)=\log s(f)=0.543535 \ldots .
$$

To be more comprehensive we plot in Figure 8 and Figure 9 the variation of the topological entropy with each of the parameters. As we can observe, there are large parameter intervals where the dynamic of the Bouali system is chaotic.

As far as the interpretation of the Bouali system is concerned, we emphasize that this firm model analyzes an asymptotic chaotic behaviour of the financial dynamics of a real firm. This chaotic state does not occur for real firms for long term but only for hours or days. The financial actions of managers break the instability and the strange atractor disappears. These managerial actions at least change the kind of instability but are unable to give a steady state growth. The model is an heuristic system. It shows the risks of some conjunction of facts leading to the chaotic attractors.

Notice that the symbolic dynamics theory, particularly the study of the kneading sequences, allows us to represent the curves, in the parameter space, corresponding to the periodic orbits of the turning point $C$. The diagram of Figure 10 shows how the periods $(n \leq 5)$ are organized throughout the parameter space considered (whose pairs of values $(s, r)$ correspond to logistic-like maps). From left to right in Figure 10, the corresponding kneading orbits are: 1-period $C^{\infty}, 2$-period - $(R C)^{\infty}, 4$-period - $(R L R C)^{\infty}, 5$-period $(R L R R C)^{\infty}, 3$-period - $(R L C)^{\infty}, 5$-period - $(R L L R C)^{\infty}$

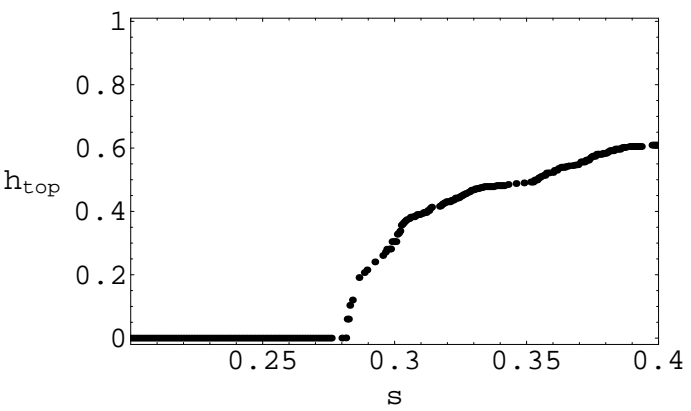

FIG. 8: Variation of the topological entropy for $s \in[0.2,0.4]$ with $r=0.068$.

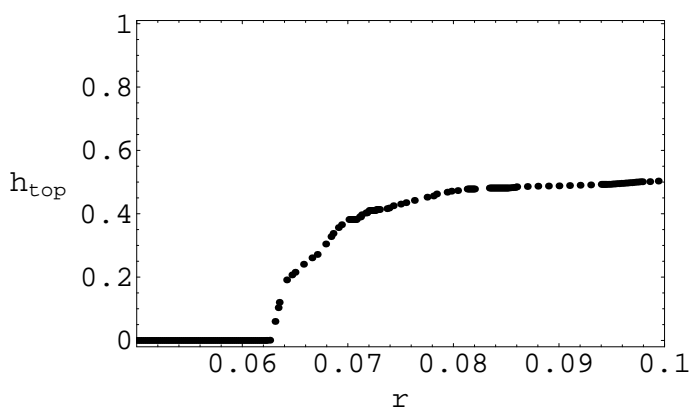

FIG. 9: Variation of the topological entropy for $r \in[0.05,0.1]$ with $s=0.3$.

and 4-period - $(R L L C)^{\infty}$. The $s r$-parameter space ordering of the kneading sequences leads to the identification of different levels for the topological entropy, which remains constant over each curve. Therefore, with this procedure, we are able to identify values of the debt rate $s$ and values of the interest rate $r$ which correspond to different levels of complexity. The following scheme represents some kneading sequences and the corresponding topological entropy.

$\begin{array}{lll}\begin{array}{l}\text { Kneading } \\ \text { sequences }\end{array} & \begin{array}{l}\text { Characteristic } \\ \text { polynomial }\end{array} & \begin{array}{l}\text { Topologica } \\ \text { entropy }\end{array} \\ R C & 1-t & 0 \\ R L R C & -1+t+t^{2}-t^{3} & 0 \\ R L R R C & -1+t-t^{2}-t^{3}+t^{4} & 0.414013 \ldots \\ R L C & -1-t+t^{2} & 0.481212 \ldots \\ R L L R C & 1-t-t^{2}-t^{3}+t^{4} & 0.543535 \ldots \\ R L L C & 1+t+t^{2}-t^{3} & 0.609378 \ldots\end{array}$

This is an example of how our understanding of the parameter space can be enhanced by the techniques of symbolic dynamics. 


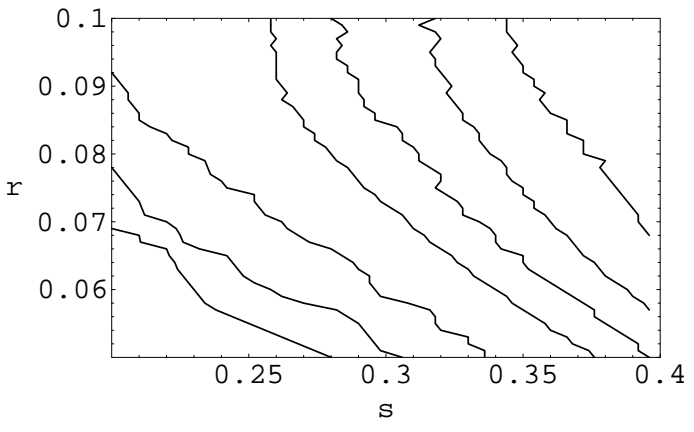

FIG. 10: Periodic orbits $(n \leq 5)$ of the turning point $C$ in the parameter region. From left to right, the corresponding kneading sequences are: $C^{\infty},(R C)^{\infty},(R L R C)^{\infty}$, $(R L R R C)^{\infty},(R L C)^{\infty},(R L L R C)^{\infty}$ and $(R L L C)^{\infty}$.

\section{CONTROL OF THE CHAOTIC FIRM MODEL}

In some practical economic situations it is desirable to obtain regular and short-time predictable behavior, particularly stable equilibrium instead of chaotic orbits. In fact, and as pointed out before, the financial managers can act over some adequate control parameters in order to break the instability and to make the chaotic scenario disappear.

We begin by pointing out that the continuous time dynamics of the firm model can be discretize via a Poincaré section, which reduces, by one unity, the dimension of the phase space. Now, we briefly describe the construction of a Poincaré map.

Consider a $n$-dimensional system $d \mathbf{x} / d t=\mathbf{f}(\mathbf{x})$. Let $V$ be a $(n-1)$-dimensional surface, called a Poincaré section. $V$ is required to be transverse to the flow. We define a Poincaré map $T$ from $V$ to itself, obtained by following trajectories from one intersection with $V$ to the next. If $\mathbf{x}_{n} \in V$ denotes the $n^{\text {th }}$ intersection, then the Poincaré map is defined by $\mathbf{x}_{n+1}=T\left(\mathbf{x}_{n}\right)$. In our particular case, we have a system with three dynamical variables $P, R$ and $F$. Since the control is usually designed for parameter values where the system is known to exhibit chaotic motion we fix, for illustrative purposes, the parameter values $r=0.068$ and $s=0.366$, where the system's attractor exhibits positive topological entropy (see Example 1). We consider a Poincaré plane of the form $F=k, k \in \mathbb{R}$, namely, $F=-0.2$ (see dashed line in Figure 3). We record the successive intersections of the trajectory with the plane, which are specified by two coordinates: $P_{n}$ and $R_{n}$. This is the discrete map we are going to consider. In the next lines we will apply the pole placement control method to the discrete Poincaré map in order to stabilize a unstable period-one orbit embedded in the chaotic attractor. By applying small adequate chosen perturbation to the dynamical system, the original chaotic trajectory can be converted into the desired stable fixed point. We consider the established fixed values for the parameters $v, m, n$ and $r$, and allow the parameter $s$ to vary in some small interval $\left|s-s_{0}\right|<\delta$, $\delta>0$, around the nominal value $s_{0}=0.366$, for which the map has a chaotic attractor.

The pole placement technique (see [18] and [1]), which is a feedback control method, extends the OGY method, allowing for a more general choice of the so called feedback matrix.

In our Poincaré surface of section $F=-0.2$ the unstable fixed point to be stabilized is located approximately at $\left(P^{*}, R^{*}\right)=(7.01449,0.306068)$. The control strategy consists in finding a stabilizing local feedback control law, which is a linear map, obtained by using least squares fitting on the sampled data in a small neighborhood of the fixed point $\left(P^{*}, R^{*}\right)$ given by

$$
\left[\begin{array}{c}
P_{t+1}-P^{*} \\
R_{t+1}-R^{*}
\end{array}\right]=A\left[\begin{array}{c}
P_{t}-P^{*} \\
R_{t}-R^{*}
\end{array}\right]+B\left(\rho-\rho_{0}\right)
$$

where

$$
A=\left[\begin{array}{cc}
0.645454 & -4.97596 \\
-0.00694097 & -0.191934
\end{array}\right], B=\left[\begin{array}{c}
4.00994 \\
0.41350
\end{array}\right]
$$

and $\left(\rho-\rho_{0}\right)$ corresponds to a parameter which is available for small perturbations to be applied to the control law (2). The ergodic nature of the chaotic dynamics ensures that the state trajectory eventually enters into the neighborhood of the fixed point. Once inside, we apply the stabilizing feedback control law in order to steer the trajectory towards the desired orbit.

Now, we verify whether the system is controllable. A system is called controllable if a matrix $K_{1 \times n}$ can be found such that $A-B K$ has any desired eigenvalues. This is possible if $\operatorname{rank}(C)=n$, where $n$ is the dimension of the state space and $C$ is the $(n \times n)$ matrix

$$
C=\left[B \vdots A B \vdots A^{2} B \vdots \ldots \vdots A^{n-1} B\right]
$$

In our case it follows that

$$
C=[B \vdots A B]=\left[\begin{array}{rlr}
4.00994 & \vdots & 0.530674 \\
\vdots & \\
0.41350 & \vdots & -0.107198
\end{array}\right]
$$

which has rank 2, and so the system is controllable. This matrix $C$ is the controllability matrix.

Assume in a small neighborhood around the fixed point $\left(P^{*}, R^{*}\right)$,

$$
\rho-\rho_{0}=-K\left[\begin{array}{c}
P_{t}-P^{*} \\
R_{t}-R^{*}
\end{array}\right]
$$

where $K=\left[\begin{array}{ll}k_{1} & k_{2}\end{array}\right]$ is a constant vector to be determined. 
The linearized map becomes

$$
\left[\begin{array}{c}
P_{t+1}-P^{*} \\
R_{t+1}-R^{*}
\end{array}\right]=[A-B K]\left[\begin{array}{c}
P_{t}-P^{*} \\
R_{t}-R^{*}
\end{array}\right],
$$

with $[A-B K]$ given by

$$
\left[\begin{array}{cc}
0.645454-4.00994 k_{1} & -4.975956-4.00994 k_{2} \\
-0.00694097-0.41350 k_{1} & -0.191934-0.41350 k_{2}
\end{array}\right],
$$

which shows that the fixed point is then stable as long as the $(2 \times 2)-$ matrix $A-B K$ is asymptotically stable, that is, all its eigenvalues have modulus less than unity.

The determination of $K$, such that the eigenvalues of the matrix $A-B K$ have specified values is called, in the theory of control systems, pole placement technique. The eigenvalues $\lambda_{1}$ and $\lambda_{2}$ of the matrix $A-B K$ are called the regulator poles, and the problem of placing these poles at the desired location, by choosing $K$ with $A$ and $B$ given, is the pole placement problem.

In our particular case, the characteristic polynomial, associated to the matrix $A-B K$, is given by

$$
\begin{aligned}
p(\lambda)= & \lambda^{2}+\left(-0.45352+4.00994 k_{1}+0.4135 k_{2}\right) \lambda+ \\
& +\left(-0.158423-1.28791 k_{1}-0.294728 k_{2}\right) .
\end{aligned}
$$

Since the eigenvalues verify the equations

$$
\begin{aligned}
& \lambda_{1} \lambda_{2}=-0.158423-1.28791 k_{1}-0.294728 k_{2} \text { and } \\
& \lambda_{1}+\lambda_{2}=-\left(-0.45352+4.00994 k_{1}+0.4135 k_{2}\right),
\end{aligned}
$$

the lines of marginal stability can be determined by solving the equations

$$
\lambda_{1}= \pm 1 \quad \text { and } \quad \lambda_{1} \lambda_{2}=1
$$

These conditions guarantee that the eigenvalues $\lambda_{1}$ and $\lambda_{2}$ have modulus less than unity for $k_{1}$ and $k_{2}$ within a certain region. This region is define by the three lines of marginal stability:

$$
\begin{aligned}
& k_{2}=-3.93048-4.36983 k_{1}, \\
& k_{2}=-3.28725-22.9181 k_{1}, \\
& k_{2}=1.82865-7.48044 k_{1} .
\end{aligned}
$$

We obtain stable eigenvalues considering $k_{1}$ and $k_{2}$ within the triangular region depicted in Figure 11. Selecting, for example, $k_{1}=0.8$ and $k_{2}=-5$ inside the triangular region, $\Omega$, and applying the control linear law (2) we obtain the desired time period-one orbit (see Figure 12 and Figure 13). At this stage it should be pointed out that depending on the values of $k_{1}$ and $k_{2}$ in the basin of attraction $\Omega$, the controlled orbit will converge towards the fixed point but takes different periods of time in order to fully accomplish the convergence process. The

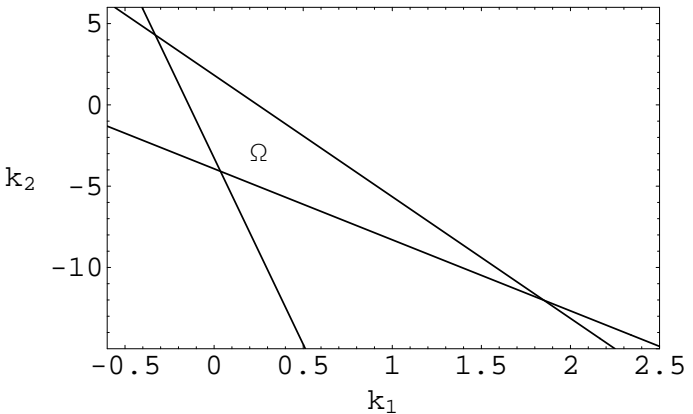

FIG. 11: The bounded region $\Omega$ that corresponds to stable regulator poles.

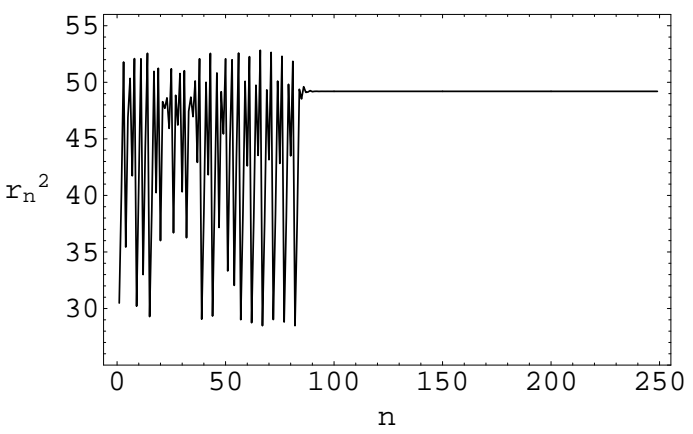

FIG. 12: Time series data for the discrete Poincaré map without and with control, setting $r_{n}^{2}=P_{n}^{2}+R_{n}^{2}$. The control is activated after the $84^{\text {th }}$ iterate.

chaotic trajectory will also converge to the desired fixed point if, in contrast, we consider fixed values of $k_{1}$ and $k_{2}$ and randomly choose some initial conditions inside the neighborhood of $\left(P^{*}, R^{*}\right)$.

Theoretically, after switching on the control, the orbit continues to perform chaotic behavior for some time, unchanged from the uncontrolled case, because it is no close enough to the fixed point. After some steps this is eliminated and the orbit is rapidly brought to the fixed point. The pole placement control strategy works very well for the chaotic firm model. In fact, our numeri-

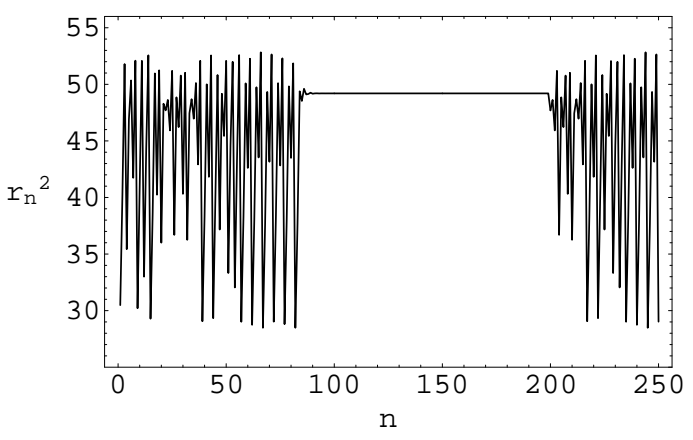

FIG. 13: Time series data for the discrete Poincaré map without and with control, setting $r_{n}^{2}=P_{n}^{2}+R_{n}^{2}$. The control is switched off after the $200^{\text {th }}$ iterate. 
cal simulations revealed a fast convergence for different initial conditions in the neighborhood of $\left(P^{*}, R^{*}\right)$ and different values of $k_{1}$ and $k_{2}$ in region $\Omega$.

\section{FINAL CONSIDERATIONS}

In this paper we have provided new insights into the study of an economic model presented by S. Bouali, which explores the dynamics of three continuous variables: profits, reinvestments and financial flow of borrowings. The extremely rich and complex behavior of this model allowed us to apply different theoretical and numerical approaches. More precisely, we analyzed the model in terms of symbolic dynamics theory and in terms of applicability of chaos control theory.

In the field of economic sciences, where comprehensive studies about chaotic attractors in terms of symbolic dynamics and measurements of complexity are rare, the use of powerful tools for the analysis of dynamic models, such as the symbolic dynamics theory, stands out to be extremely effective for the computation of an important numerical invariant related to the exponential orbit growth - the topological entropy. In fact, the introduction and rigorous study of the iterated one-dimensional maps related to the profits motion, that incorporate the salient dynamical properties of the system, became possible by analyzing the variation of this measure of complexity with the two control parameters $s$ and $r$. Our analysis reveals that when the debt rate $s$ (proportional to selffinancing) and the interest rate $r$ increase the topological entropy starts to be zero and once has accomplished a positive value begins to increase. Therefore, high values of these control parameters tend to induce more complexity to the model.

The representation of the isentropic curves (corresponding to the periodic orbits of the turning point $C$ ) in the $s r$-parameter space allowed us to introduce the parameter space ordering of the dynamics. In fact, this construction gives insights about the behavior of the topological entropy in all the parameter space considered.

Indeed, the family of maps associated with the motion of profits exhibits positive topological entropy, which demonstrates its chaotic nature. The techniques of symbolic dynamics allowed us to quantify the orbit complexity and to distinguish different chaotic regimes, extracting order from chaos, in a significant region of the parameter space.

Motivated by the chaotic structure of the model and the central role of regular cycles in economy, we have applied the pole placement control method in order to obtain predictable behavior - the stabilized period-one orbit. In a real context, it is meaningful to choose a control parameter that can be directly influenced by the financial managers of the firm. Therefore, we have taken the debt rate $s$ as the control parameter and we showed, numerically, that the complicated motion that emerges from the dynamics of the model can be controlled by small parameter perturbations (which corresponds to the possible actions of the financial managers) in a control linear law deduced from a Poincaré section. The fundamental characteristics of the model are not changed by the control procedure as the fixed point, that forms the basin of attraction, remains the same. We emphasize that, with the application of the chaos control technique, the model performs fast convergence for different initial conditions and different values of the control parameters. The chaotic dynamics could be converted, by using just a small feedback control, to motion on a desired period orbit.

\section{Acknowledgements}

The authors would like to thank Professor Safieddine Bouali for his enlightenments and valuable information. The financial support from the Fundação Ciência e Tecnologia, Lisbon, is gratefully acknowledged by the third author, under the contracts No PTDC/GES/73418/2006 and No PTDC/GES/70529/2006.

\section{References}

[1] Romeiras F J, Grebogi C, Ott E and Dayawansa W P 1992 Physica D $\mathbf{5 8} 165$

[2] Day R H 1996 Complex Economic Systems (MIT Press)

[3] Grandmont J M 1985 Econometrica 53995

[4] Gleik J 1988 Chaos: Making a New Science (Viking Penguin, New York)

[5] Katok A, Hasselblatt B 1999 Introduction to the Modern Theory of Dynamical Systems (Cambridge University Press)

[6] Bouali S 2002 The Hunt Hypothesis and the Dividend Policy of the Firm. The Chaotic Motion of the Profits e-print arXiv: nlin.CD/0206032
[7] Ott E, Grebogi C and Yorke J A 1990 Phys. Rev. Lett. 641196

[8] Mendes Diana A and Mendes Vivaldo 2005 Journal of Physics: Conference Series 23158

[9] Milnor J and Thurston W 1988 Lect. Notes in Math. 1342465 (Springer-Verlag)

[10] Lampreia J P and Sousa Ramos J 1997 Portugal. Math. 541

[11] Hao Bai-Lin and Zheng Wei-Mou 1998 Applied symbolic dynamics and chaos. Directions in Chaos $\mathbf{7}$ (World Scientific Publishing Co)

[12] Glasner E and Weiss B 1993 Nonlinearity 61067 
[13] Blanchard F. 2008 Topological chaos: what may this mean? arXiv:0805.0232v1 [math.DS].

[14] Gilmore R. and Lefranc M 2002 The topology of chaos (Wiley-Interscience, John Wiley \& Sons, Inc, New York).

[15] Ruette S. Chaos for Continuous Interval Maps - A survey of relationship between the various sorts of chaos, preprint.
[16] Lampreia J P and Sousa Ramos J 1989 European Conference on Iteration Theory 431 (World Sci. Publishing)

[17] Misiurewicz M and Szlenk W 1980 Studia Math. 6745

[18] Ogata K 1990 Modern Control Engineering (New Jersey, Prentice-Hall) 